\title{
Performance of Fast Cell Selection in Two-Tier OFDMA Networks with Small Cells
}

\author{
Zdenek Becvar, Pavel Mach \\ Department of Telecommunication Engineering, Faculty of Electrical Engineering, Czech Technical University in Prague \\ Technicka 2, 16627 Prague, Czech Republic \\ zdenek.becvar@fel.cvut.cz, machp2@fel.cvut.cz
}

\begin{abstract}
If large amount of small cells, i.e., femtocells, picocells or microcells, is deployed, users perform handover among small cells and macrocells very often. In OFDMA networks, only hard handover is usually supported as this type can be implemented easily. On the other hand, frequent hard handover negatively influence a quality of service experienced by users. The degradation of the quality is a consequence of a short interruption in communication during hard handover and due to redundant overhead generated for controlling and managing the handover procedure. This paper investigates performance of a fast cell selection applied in two-tier networks with small cells and compares it with the hard handover. We focus on the impact of a backhaul capacity limitation on the performance of both hard handover and fast cell selection. As our results indicate, there is a gain in throughput introduced by the fast cell selection if the backhaul of the small cells is not a bottleneck in term of capacity. Nevertheless, if the backhaul capacity is limited, the fast cell selection is profitable only for low bitrates. This observation indicates a need for mandatory consideration of the backhaul capacity for the fast cell selection in the networks with small cells.
\end{abstract}

Keywords-fast cell selection; hard handover; small cells; mobility management; performance evaluation

\section{INTRODUCTION}

The $4 \mathrm{G}$ mobile networks are assumed to be deployed at frequencies in order of $\mathrm{GHz}$ (e.g., 2 or $2.6 \mathrm{GHz}$ ). To cover potential gaps in coverage due to heavy attenuation of a signal at higher frequencies, small cells can be deployed. In general, two types of the small cells are distinguished: femtocells and pico/microcells. In both cases, radius of the small cells is low (i.e., in order of tens of meters). The femtocells are assumed to be placed in users' premises or enterprises, owned by users and also controlled by users. Their connection to the core network is enabled via a backhaul of limited capacity and variable quality (typically, ADSL is used). The femtocells can provide open access, closed access, or their combination - hybrid access. In the case of the open access, all users can enter the femtocell. Contrary, only users included in a Closed Subscribed Group (CSG) list are admitted to the closed femtocell. The pico/microcells can be also deployed in users' premises; however, these cells are supposed to be deployed mostly in enterprises or at public places [1]. Pico/microcells are under full control of an operator and should be interconnected with an operator's backbone by a high quality link.

Dense deployment of the small cells introduces new challenges related especially to an interference mitigation for the closed access and to a user's mobility management for the open or hybrid access [2][3]. A mobile user is forced to perform handover from a serving cell to a target cell to keep the quality of service (QoS). If a user is moving close to the open access small cells, large number of handovers can be performed during short time interval. Hence, a significant drop in QoS is introduced due to the hard handover. The amount of handovers can be adjusted by techniques used for elimination of redundant handovers, such as a hysteresis or a delay timer. Unfortunately, these techniques considerably decrease user's throughput in networks with small cells [4]. Moreover, an interruption is still observed if a conventional hard handover is performed as the user is disconnected from the serving cell before a new connection to the target cell is established.

Fast Cell Selection (FCS) can be exploited instead of the hard handover to suppress the handover interruption and QoS decrease. However, an implementation of FCS to real networks is more demanding and more complex comparing to the hard handover. In the case of FCS, so-called Active Set (AS) is defined for each User Equipment (UE). The AS is comprised of several neighboring cells of the UE. Neighbor cells are added/removed to/from the AS depending on the signal level measured by the UE [5]. Improvements of the conventional FCS can be found, for example, in [6][7]. These papers show an increase in throughput by FCS. Nevertheless, all the papers are focused on the networks with macrocells only.

The contribution of this paper consists in investigation of the throughput of a conventional hard handover and FCS in the networks with small cells providing open or hybrid access. Closed access is not considered since only a limited amount of CSG users (typically up to four users per a small cell [8]) is admitted to these small cells. We also investigate the impact of the backhaul capacity of the small cells on the performance of the hard handover and FCS.

The rest of the paper is organized as follows. The next section defines a model and simulation scenario for evaluation of the performance. Further, Section III presents simulation results and provides discussion of the results. In the last section, major conclusions are summarized.

\section{SYSTEM MODEL}

From the performance evaluation point of view, a difference between the femto and pico/microcells consists in the capacity of the small cell backhaul. To avoid any 
ambiguities in terminology, we use the "small cell" with the meaning of the "femtocell" if the backhaul is limited and the label "pico/microcell" if the backhaul is unlimited. By unlimited backhaul is understood the backhaul able to serve all radio traffic, that is, if the backhaul capacity is higher than the radio capacity. In the simulations, "unlimited" backhaul is represented by the backhaul capacity of $100 \mathrm{Mbps}$.

For the evaluation, a rural scenario with fifty randomly deployed houses within a macrocell is considered according to the recommendations defined by Small Cell Forum [9]. All houses are of a square shape with a size of 10x10 meters as depicted in Fig. 3. Each house is equipped with one randomly deployed small cell and occupied by one indoor UE. The indoor UE moves in line with the probabilistic waypoint mobility model based on [10]. For this model, several points of stay and a point of decision are defined. In the point of decision, the indoor UE selects a point of stay with equal probability for all points. The time spent in the point of stay is generated according to the normal distribution taken over from [10]. Beside indoor UEs, also one hundred outdoor UEs are randomly dropped in the simulation area. All outdoor UEs follow the probabilistic random walk mobility model with a speed of $1 \mathrm{~m} / \mathrm{s}$.

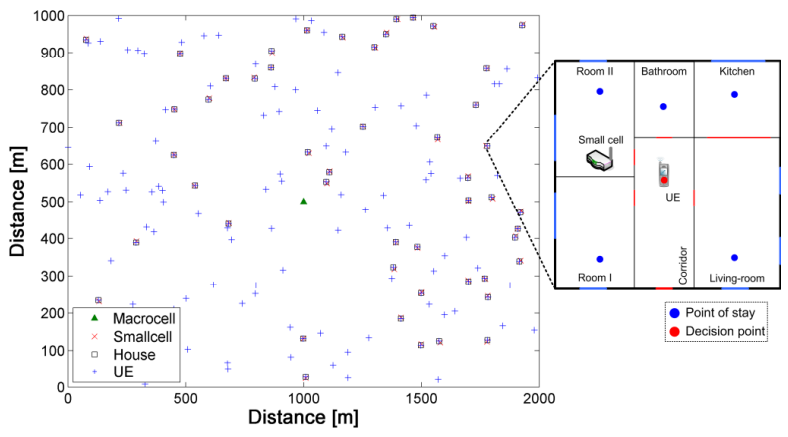

Figure 1. Simulation deployment and model of a house

Channel models are also based on recommendations of Small Cell Forum presented in [9]. The path losses are modeled according to ITU-R P.1238 and Okumura-Hata for communication with small cells and macrocells respectively. The major transmission, channel, and simulation parameters are summarized in Table I.

TABLE I. SIMULATION PARAMETERS

\begin{tabular}{|l|l|}
\hline \multicolumn{1}{|c|}{ Parameter } & \multicolumn{1}{c|}{ Value } \\
\hline Frequency band [GHz] & 2 \\
\hline Channel bandwidth for macro/small cell [MHz] & $20 / 20$ \\
\hline Transmitting power of macro/small cell [dBm] & $46 / 15$ \\
\hline Height of macro/small cell/UE [m] & $32 / 1 / 1.5$ \\
\hline Std. deviation of shadowing of MBS/FAP [dB] & $8 / 4$ \\
\hline Loss of outer/inner walls [dB] & $10 / 5$ \\
\hline Noise density [dBm/Hz] & -174 \\
\hline LTE-A physical layer overhead [\%] & 25 \\
\hline Number of macro/small cells & $1 / 50$ \\
\hline Number of indoor/outdoor UEs & $50 / 100$ \\
\hline Number of simulation drops & 10 \\
\hline Duration of a simulation drop [s] & 7200 \\
\hline
\end{tabular}

For data transmission, TDD LTE-A physical layer with UL-DL configuration " $l$ " and special subframe configuration " $O$ " is implemented. Each user (outdoor as well as indoor) offers constant bit rate traffic during the whole simulation. User's data are served in such manner that the bandwidth is fairly allocated to provide the same throughput for all users. For the open access, indoor as well as outdoor users share the radio resources and the backhaul of the small cell with equal priority. On the other hand, for the hybrid access, a half of the radio and backhaul capacity is reserved for the indoor UEs. Then all outdoor UEs share the rest of the available capacity.

The performance evaluation is presented through served throughput for indoor, outdoor, and cell edge users. Served throughput represents the amount of really transferred user data. It is observed for indoor, outdoor, and cell edge users. The indoor users are all users located inside the houses (50 indoor UEs in the simulations) while the outdoor users are all other users (100 UEs in our simulations). The cell edge UEs are the users positioned close to the border of two neighboring cells. According to [7], we define the cell edge UE as the user with the level of the signal from the second strongest cell $\left(s_{2}\right)$ within the $T_{\text {cell_edge }}$ threshold (in simulations, equal to $1 \mathrm{~dB}$ ) from the signal level of the strongest cell (serving, $s_{s}$ ) as defined in the subsequent equation:

$$
s_{s}-s_{2}<T_{\text {cell_edge }}
$$

An amount of the cell edge users varies in time depending on the users' location. Nevertheless, the trajectories of the UEs are the same for the evaluation of both hard handover and FCS. Thus, the amount of the cell edge UEs is the same for both as well.

\section{Simulation RESUlts}

This section presents performance of the hard handover and FCS obtained by simulations in MATLAB.

The served throughput over the level of traffic offered by individual types of UEs is depicted in Fig. 6 - Fig. 8. Each figure consists of two subplots showing average throughput for open (left plots) and hybrid (right plots) accesses. In addition, all figures contain results for the backhaul with limited capacity of $8 \mathrm{Mbps}$ (solid lines) and an unlimited backhaul with the capacity of $100 \mathrm{Mbps}$ (dashed lines).

The results confirm the fact that an increase in $\Delta_{H M}$ for the hard handover lowers the throughput. This is caused by keeping UEs connected to the serving cell for a longer time even if the target cell is able to provide a channel with higher quality. For FCS, an impact of the thresholds depends on the type of the access and the backhaul capacity. For the unlimited backhaul, throughput increases with $T_{\text {add }}$ and $T_{\text {del }}$ for the outdoor UEs as more cells are included in the AS and interference experienced by the outdoor UEs is lowered. For the indoor UEs served by the open access cells with the unlimited backhaul, the throughput is limited by the backhaul and a positive impact of increase in $T_{a d d}$ and $T_{d e l}$ is negligible. If the backhaul is limited, higher $T_{a d d}$ and $T_{d e l}$ decrease the throughput of the indoor UEs in the case of the open access. It 
is a cost of sharing the backhaul with more outdoor UEs who experience slight increase in their throughput. Nevertheless, this rise in throughput of the outdoor UEs is limited by the backhaul capacity. For the hybrid access with the limited backhaul, an impact incurred by $T_{a d d}$ and $T_{d e l}$ is negligible due to the fixed allocation of the resources among indoor and outdoor UEs.

According to Fig. 6, FCS is profitable for the indoor UEs if a sufficient backbone capacity (100Mbps) is provided for both the open and hybrid accesses. If the backhaul is of a limited capacity (8 Mbps), FCS introduces heavy loss in throughput of the indoor UEs for the open access. This loss is a result of fair sharing the small cell backhaul capacity with the outdoor UEs. If the small cell provides higher channel quality than the macrocell, each UE is trying to transmit data via the small cell, but the backhaul is not able to serve the data. The hybrid access with the limited backhaul reaches the same performance for both FCS and hard handover since the fixed ratio of the backhaul capacity is reserved for the indoor UEs.

Performance of the outdoor UEs is influenced in more positive way by FCS (see Fig. 7). Again, FCS is profitable for all levels of the offered traffic and both accesses if the small cell backhaul is unlimited. For the limited backhaul, FCS increases throughput for offered traffic up to 2 and $1.5 \mathrm{Mbps}$ for the open and hybrid accesses respectively. Again, the gain of the hard handover for high level of the traffic and the limited backbone is caused by sharing the resources with more UEs in the case of FCS.

Throughput of the most critical set of user's, the cell edge UEs, is depicted in Fig. 8. The set of cell edge users mostly consists of the outdoor UEs; thus, the behavior of the throughput of the cell edge UEs follows the results for the outdoor UEs. Therefore, FCS is profitable if a small cell is connected via unlimited backhaul. If the backhaul capacity is limited, FCS outperforms the hard handover only for lower offered traffic like in the case of the outdoor UEs.
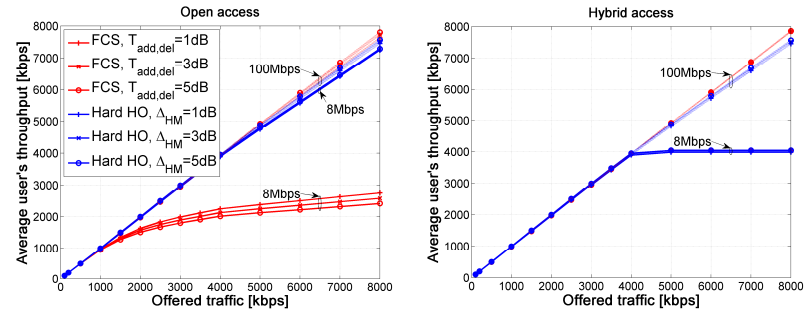

Figure 2. Served throughput of indoor UEs for open and hybrid acceses
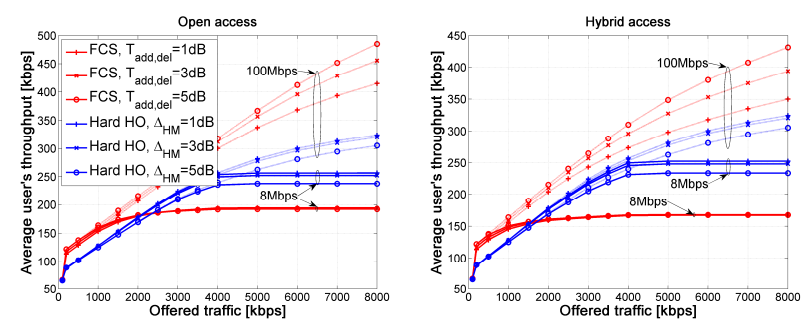

Figure 3. Served throughput of outdoor UEs for open and hybrid acceses
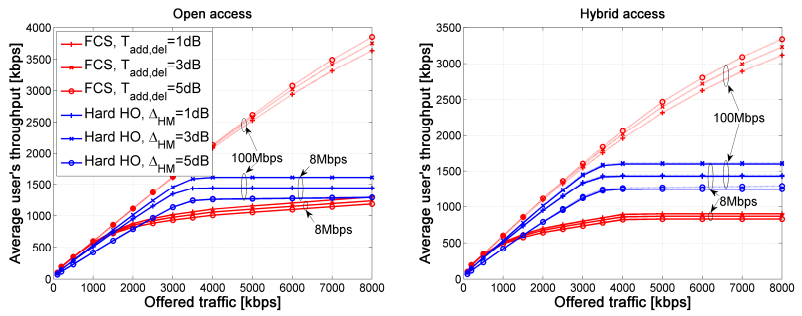

Figure 4. Served throughput of cell edge UEs for open and hybrid acceses

\section{CONCLUSIONS}

In this paper, we investigated performance of FCS and the hard handover if small cells, connected to the network via either limited or unlimited backhaul, are considered. The results show FCS introduces significant gain for all UEs for the unlimited backhaul capacity, i.e., for pico/microcells. On the other hand, the throughput is improved by FCS only for the outdoor UEs offering low throughput up to $2 \mathrm{Mbps}$ if the backhaul capacity is limited (i.e., for femtocells). Therefore, if the small cells are deployed, the conventional FCS can even decrease performance in term of the throughput if the backhaul capacity is not considered in the active set management. Hence, the conventional algorithms for mobility support should be aware of available capacity of the small cell backhaul to maximize throughput of users.

\section{ACKNOWLEDGMENT}

This work has been supported by Grant no. P102/12/P613 funded by the Czech Science Foundation.

\section{REFERENCES}

[1] Small Cell Forum whitepaper, " Small cells - what's the big idea? Femtocells are expanding beyond the home," February 2012

[2] V. Chandrasekhar, J. Andrews, A. Gatherer, "Femtocell Networks: A Survey," IEEE Communication Magazine, vol.46, no. 9, June 2008.

[3] G. de la Roche, A. Valcarce, D. Lopez-Perez, J. Zhang " Access control mechanisms for femtocells," IEEE Communication Magazine, vol.48, no. 1 , pp. 33-39, January 2010.

[4] T. Jansen, I. Balan, J. Turk, I. Moerman, and T. Kurner, "Handover parameter optimization in LTE self-organizing networks," IEEE VTC 2010-Fall, September 2010.

[5] 3GPP Technical Specification TS 25.433 v3.14.2 "UTRAN Iub interface NBAP signalling," Septemeber 2004.

[6] H.H. Choi, J.B. Lim, H. Hwang, and K. Jang, "Optimal Handover Decision Algorithm for Throughput Enhancement in Cooperative Cellular Networks," IEEE VTC 2010- Fall, Ottawa, September 2010.

[7] M. Feng, X. She, L. Chen , and Y. Kishiyama, " Enhanced Dynamic Cell Selection with Muting Scheme for DL CoMP in LTE-A," IEEE VTC 2010-Spring, May 2010.

[8] V. Chandrasekhar, J.G. Andrews, and A. Gatherer, "Femtocell Networks: A Survey," IEEE Communication Magazine, vol. 46, no. 9, pp. 59-67, September 2008.

[9] Femto Forum whitepaper, "Interference managemetn in OFDMA femtocells," March 2010.

[10] H. Claussen, L.T.W. Ho, and L.G. Samuel, "Self-optimization of Coverage for Femtocell Deployment," IEEE Wireless Telecommunication Symposium WTS 2008, pp. 278-285, April 2008. 